Final Technical Report for

DOE Project:

Beam Stripping Solutions for RIA

Colorado School of Mines

P.I.: Dr. Uwe Greife, Associate Professor

DE-FG02-03ER41242 


\section{Possibilities for Beam Stripping Solutions at a Rare Isotope Accelerator (RIA)}

Faculty:

Uwe. Greife*

Graduate Students:

Ellen Simmons (graduated 2005 with a M.Sc. from this project), Luke. Erikson, Cybele Jewett, Jake Livesay and Kelly Chipps

Department of Physics, Colorado School of Mines, Golden, Colorado, USA

(*) corresponding author: Department of Physics, Colorado School of Mines, 1523 Illinois

Street, Golden, CO 80401, USA; phone: (303) 273 3618; fax: (303) 273 3919; email:

ugreife@mines.edu

Executive Summary

As part of the DOE RIA R\&D effort we investigated the possibilities and problems of beam strippers in the different heavy ion accelerator components of a possible Rare Isotope Accelerator (RIA) facility. We focused on two beam stripping positions in the RIA heavy ion

driver where benchmark currents of up to 5 particle $\mu \mathrm{A}{ }^{238} \mathrm{U}$ were projected at energies of 10.5 $\mathrm{MeV} / \mathrm{u}$ and $85 \mathrm{MeV} / \mathrm{u}$ respectively. In order to select feasible stripper materials, data from experiments with Uranium beams at Texas A\&M and GSI were evaluated. Based on these results 
thermal estimates for a possible design were calculated and cooling simulations with commercially available software performed. Additionally, we performed simulations with the GEANT4 code on evaluating the radiation environment for our beam stripping solution at the 85 $\mathrm{MeV} / \mathrm{u}$ position in the RIA driver.

\section{Introduction}

The most recent concept for the RIA accelerator complex foresaw two beam-stripping positions in the driver accelerator $[1,2]$. This design is optimized for accelerating high power uranium beams starting from an ECR ion source at charge state 29. To keep the total accelerating voltage relatively low, two stripping stages are assumed, the first at $\sim 10.5 \mathrm{MeV} / \mathrm{u}$ and the second at $\sim 85 \mathrm{MeV} / \mathrm{u}$ for uranium ions. For the final design goal of $400 \mathrm{~kW}$ of uranium beam at 400 $\mathrm{MeV} / \mathrm{u}$, the beam current at the first stripper location will be $\sim 5$ particle $\mu \mathrm{A}$. In order to increase efficiency several charge states are to be accelerated after each stripping position, requiring similar current stability also from the second stripper.

In all stripping stages, the use of solid materials is preferred compared to gaseous materials, as a multitude of measurements [3] show that solid strippers achieve the higher average charge state distribution. In order to keep energy and angle straggling low, low Z materials, just thick enough to achieve charge state equilibrium, are preferred. In many energy regimes lower $\mathrm{Z}$ materials also lead to higher average charge state distributions [4]. In the area around the stopping power maximum, carbon foils have been used as target backing for experiments at GSI searching for super heavy elements with incident ion beam currents up to $0.5 \mathrm{p} \mu \mathrm{A}$ of $5 \mathrm{MeV} / \mathrm{u}$ Nickel ions [5]. In order to achieve reasonable target lifetimes, several foils are here mounted on a rotating target 
wheel. From the current data on the benchmark Uranium beam it is not clear if a foil stripper could withstand the necessary $5 \mathrm{p} \mu \mathrm{A}$ beam current at $9 \mathrm{MeV} / \mathrm{u}$ which is still near the region of the stopping power maximum. At higher beam energies $(\sim 85 \mathrm{MeV} / \mathrm{u})$ in the driver the deposited energy is lower by about a factor 2. As an alternative to solid stripper materials, Argonne National Laboratory has started the development of liquid Lithium strippers [6].

In the following we describe some of the efforts of our group to provide data and simulations to explore the technical viabilities of the different proposed stripper solutions.

\section{Measured charge state probabilities at RIA energies}

An experiment was performed at the Texas A\&M cyclotron facility with a $10.5 \mathrm{MeV} / \mathrm{u}{ }^{238} \mathrm{U}$ beam impinging on a stripper foil and the emerging charge states being measured by the MDM separator and a multi-wire chamber. The stripping foils in the Texas A\&M experiment were Carbon, Beryllium evaporated on Carbon and a thick Beryllium foil. Our analysis shows that the lighter element produces the charge state distribution with the higher centroids. The widths of the distributions are comparable for all stripping foils leaving the advantage for the lighter element also for the anticipated simultaneous transport of 5 charge states after this stripper position. If 5 charge states (70-74) can be transported, about $75 \%$ of the incident beam can be further accelerated (Fig.1). The equilibrium charge state distribution of $10.5 \mathrm{MeV} / \mathrm{u}{ }^{238} \mathrm{U}$ through Carbon foils is well described by the semi-empirical formula of Baron et al. [7]. For Beryllium foils though, the formula of Leon et al. [4] seems to underestimate the real distribution by about two charge states. As straggling seemed to be dominated in all foils by thickness variations (10$20 \%)$, numbers on energy straggling could not be extracted. 
In December 2003 an experiment was performed at GSI with our participation to determine parameters similar to the Texas A\&M experiment at $85 \mathrm{MeV} / \mathrm{u}$ Uranium beam energy. A $73^{+}$ Uranium beam was selected with the first half of the FRS separator and guided onto a selection of Carbon and Beryllium foils. The second half of the FRS dispersed the different charge states onto the focal plane where they were detected by a multi-wire chamber. Directly from the raw data it was obvious that the Carbon foils from some inexpensive suppliers had to be eliminated as possible candidates due to significant density and thickness variations. One foil type produced by Panasonic seems to be a viable solution and was further investigated. We also analyzed Beryllium foils as we expected to achieve higher charge state distributions with the lighter elements. However, adding up 4 charge states, as it is planned to further accelerate 4 charge states after this stripper position, the Carbon foil produces the more advantageous charge state distribution as a sum of four higher charge states (87-90) (compared to 86-89 in the Beryllium case) can be used in achieving larger than $80 \%$ probability (Fig. 2). As the density variations in the Carbon foils produced energy straggling that was not satisfactory, in a follow-up experiment at NSCL (MSU) also a Vanadium foil was studied [6]. As this foil also produced an agreeable charge state distribution with reduced (density variation dominated) straggling, metal foils of higher $\mathrm{Z}$ have now to be regarded as possible stripper materials and were therefore included in our temperature calculations.

III. Thermal simulations of a gas cooled Carbon foil stripper

Extensive thermal calculations using the Mathematica software have been performed by our group [8], which included thermal radiation into the surrounding, thermal conductivity through 
the axis of the wheel, as well as thermal conduction and convection through a cooling gas (Helium). The wheel simulated had an ion beam (of $1 \mathrm{~cm}$ diameter) traverse the foils mounted around $100 \mathrm{~cm}$ circumference moving with rotations up to $10 \mathrm{~Hz}$. The $1 \mathrm{~cm}$ beam diameter might need to be realized at RIA by scanning the beam over the foil [6].

Our simulations showed the principal viability of this approach for both Carbon (at 10.5 and 85 $\mathrm{MeV} / \mathrm{u}$ ) and Titanium (at $85 \mathrm{MeV} / \mathrm{u}$ ). Additional simulations with the CosmosWorks software package also showed that the principal cooling mechanism is thermal radiation. Conduction through the wheel and axis as well as the influence of the cooling gas lower the maximum temperature on the beam spot only by about $100 \mathrm{~K}$ each. Without both mechanisms for reasonable rotation speeds the foils still remain with their temperature comfortably below the melting point. Therefore, we think at this point that gas cooling will not be necessary. This will lead to a significant simplification of the stripper setup and avoid gas flow into the neighboring accelerator components. Care will though have to be taken to provide significant cooling to all surfaces surrounding the foil wheel.

In conclusion, at $10.5 \mathrm{MeV} / \mathrm{u}$ charge state distribution centroids and widths favor the use of light stripper materials. Argonne's liquid Lithium stripper, if it can be made to work, seems to be the preferred stripper solution at this station. A backup solution would be the use of a rotating Carbon wheel stripper. However, our design studies show that it will be very difficult to construct, operate and maintain a system like this at these energies due to the small thickness of the foils $\left(\sim 500 \mu \mathrm{g} / \mathrm{cm}^{2}\right)$. At $85 \mathrm{MeV} / \mathrm{u}$ it seems that the use of Carbon or even higher $\mathrm{Z}$ metal foils will be more advantageous than Beryllium and probably also liquid Lithium, leading to a preferred use of a rotating foil wheel. 
IV. GEANT4 simulations of the radiation environment

In order to provide data for area classification and shielding considerations we have investigated the possibility of describing the radiation environment around the second stripper position by using simulations of nuclear reactions in the stripper foils. Reaction products could also serve as a diagnostic monitoring the integrity of stripping foils. Taking advantage of the new capabilities of GEANT4 with the inclusion of the IONMARSE code (heavy ion reactions) we simulated the impact of $85 \mathrm{MeV} / \mathrm{u}{ }^{208} \mathrm{~Pb}$ ions on a Titanium foil. In the geometry we include beam lines and target chamber surrounded by water subjects to look for energy deposited related to radiation exposure as well as metal subjects to look for activation. Fig. 3 shows for example the total energy deposit in each of the water subjects $(15 \mathrm{~cm} \times 15 \mathrm{~cm} \times 10 \mathrm{~cm}$; at a distance of 1 meter from the stripping foil) as a function of angle using $10^{9}$ incoming ${ }^{208} \mathrm{~Pb}$ ions. Converted into absorbed dose from a $10 \mathrm{mg} / \mathrm{cm}^{2}$ Titanium stripper foil we get $3.7 \mathrm{mGy} / \mathrm{s}(0.37 \mathrm{rad} / \mathrm{s})$ under 10 degrees to the beam direction and $34 \mu \mathrm{Gy} / \mathrm{s}(3.4 \mathrm{mrad} / \mathrm{s})$ under a 170 degree backward angle. All equipment necessary to operate the stripper will have to withstand these doses and preferably needs to be installed under backward angles.

\section{Summary and Outlook}

With the macroscopic problems like cooling seemingly under control, attention has to shift to the microscopic impact the ion beam has on the stripper foils. In order to get an idea of the orders of magnitude involved we performed a quick calculation of the radiation dose that the foil receives in our wheel setup following the equations in Lee [9]. Just taking into account the linear energy 
transfer due to electronic stopping $(18.5 \mathrm{keV} / \mathrm{nm})$ results in a surprisingly high radiation dose of 170 GigaGy/day. Doing a literature search we found though no publication, which could explain us what influence this dose could have on the foils. Another way to estimate possible damage is to estimate the displacement per atom (dpa). Again a formula out of Lee [9] was used and we arrive at a dpa $=0.52$ per day for our benchmark situation. As Titanium is considered as a vessel material for fusion reactors some publications $[10,11,12]$ on material properties after light ion irradiation exist. These show significant changes (hardening and microstructure) in material properties starting around $0.2 \mathrm{dpa}$. This dpa value would already be reached in half a day at RIA. Further simulations and preferably test measurements with strongly focused beams seem to be advisable.

Acknowledgements

We thank Jerry Nolen from ANL for many enlightening discussions. He also provided us with the raw data of the Texas A\&M experiment. The data at GSI was taken during an experimental campaign headed by Maurizio Portillo from MSU.

\section{Bibliography}

[1] K.W. Shepard et al., Proc. $9^{\text {th }}$ Workshop on RF Superconductivity, Santa Fe (1999)

[2] P.N. Ostroumov, Proc. 9th International Conf. On Heavy Ion Accelerator Technology (2002) 1

[3] H.-D. Betz, Rev. Mod. Phys 44 (1972) 465 
[4] A. Leon et al. Atomic Data and Nucl. Data Tables 69 (1998) 217

[5] H. Folger et al., Nucl. Instrum. Meth. A 362 (1995) 64

[6] J. Nolan, private communication (2002)

[7] E. Baron et al., Nucl. Instrum. Meth. A 328 (1993) 177

[8] E. Simmons, M.S. Thesis, Colorado School of Mines (2005)

[9] E.H. Lee, Nucl. Instrum. Meth. B151 (1999) 29

[10] K. Nakata et al., J. Nucl. Mat. 240 (1997) 221

[11] P. Marmy and T.Leguey, J. Nucl. Mat. 296 (2001) 155

[12] T. Leguey at al., J. Nucl. Mat. 307-311 (2002) 696 
Figure 1: Sum of highest five charge state fractions (which could be transmitted in the RIA driver) emerging from different foil types and thicknesses as measured with a $10.5 \mathrm{MeV} / \mathrm{u}$ Uranium beam at Texas A\&M.

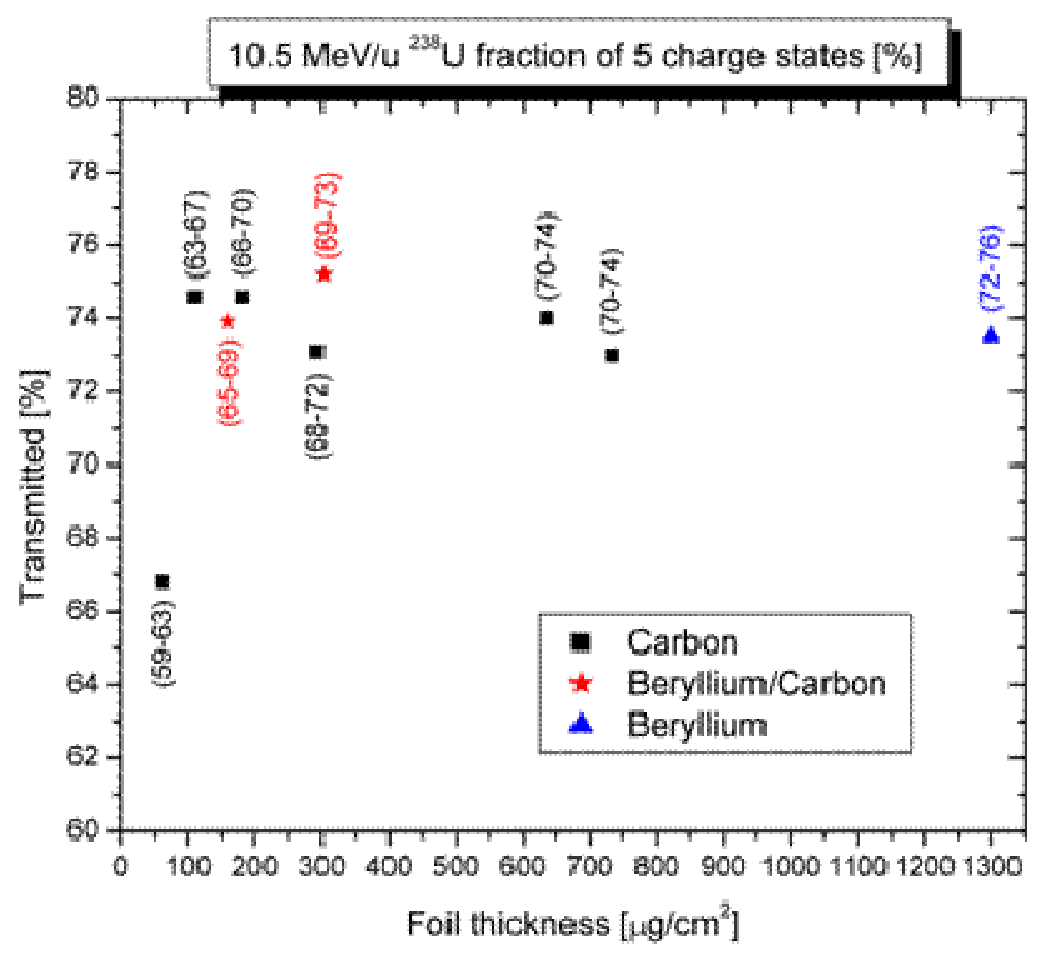


Figure 2: Sum of highest four charge state fractions (which could be transmitted in the RIA driver) emerging from different foil types and thicknesses as measured with an $85 \mathrm{MeV} / \mathrm{u}$ Uranium beam at GSI.

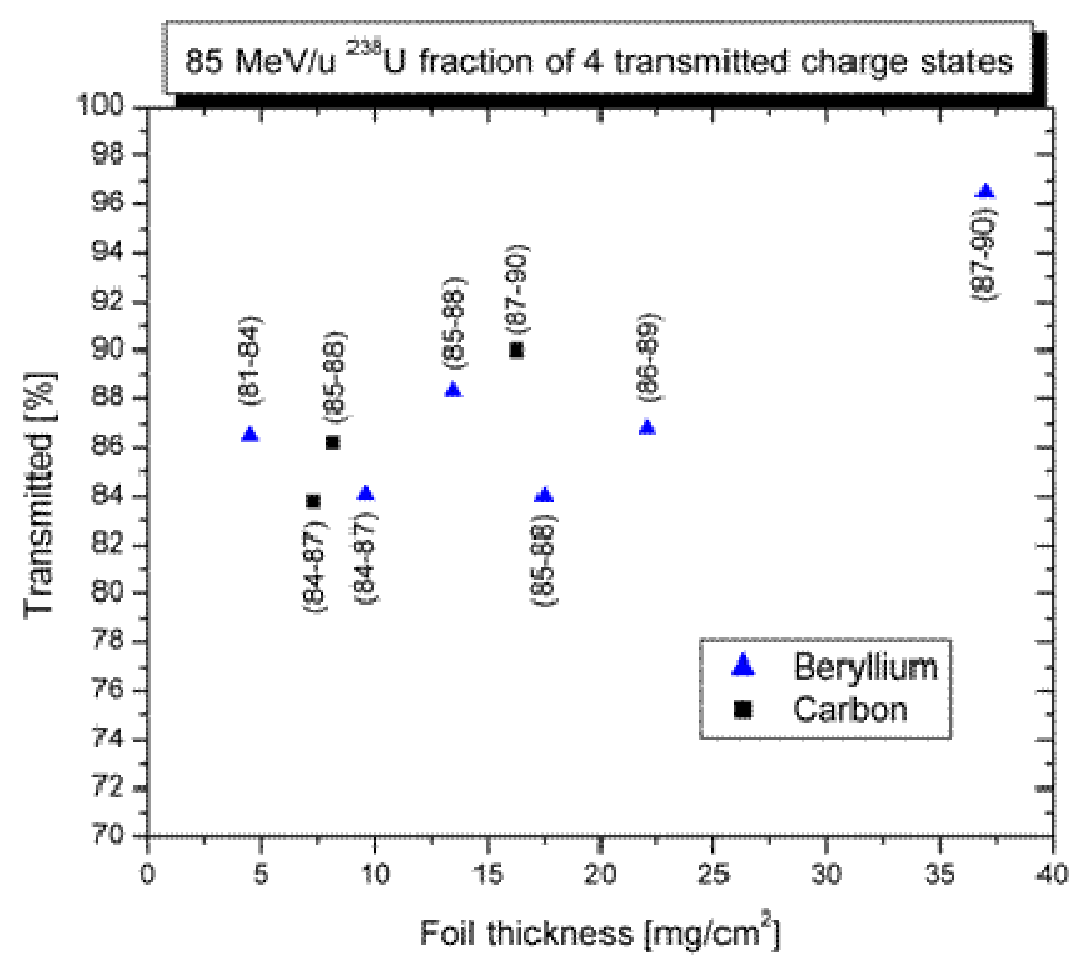


Figure 3: GEANT4 simulation of energy deposited in water objects at 1 meter distance around a $4.5 \mathrm{mg} / \mathrm{cm}^{2}$ Titanium stripper foil bombarded with $10^{9} 85 \mathrm{MeV} / \mathrm{u}^{208} \mathrm{~Pb}$ ions.

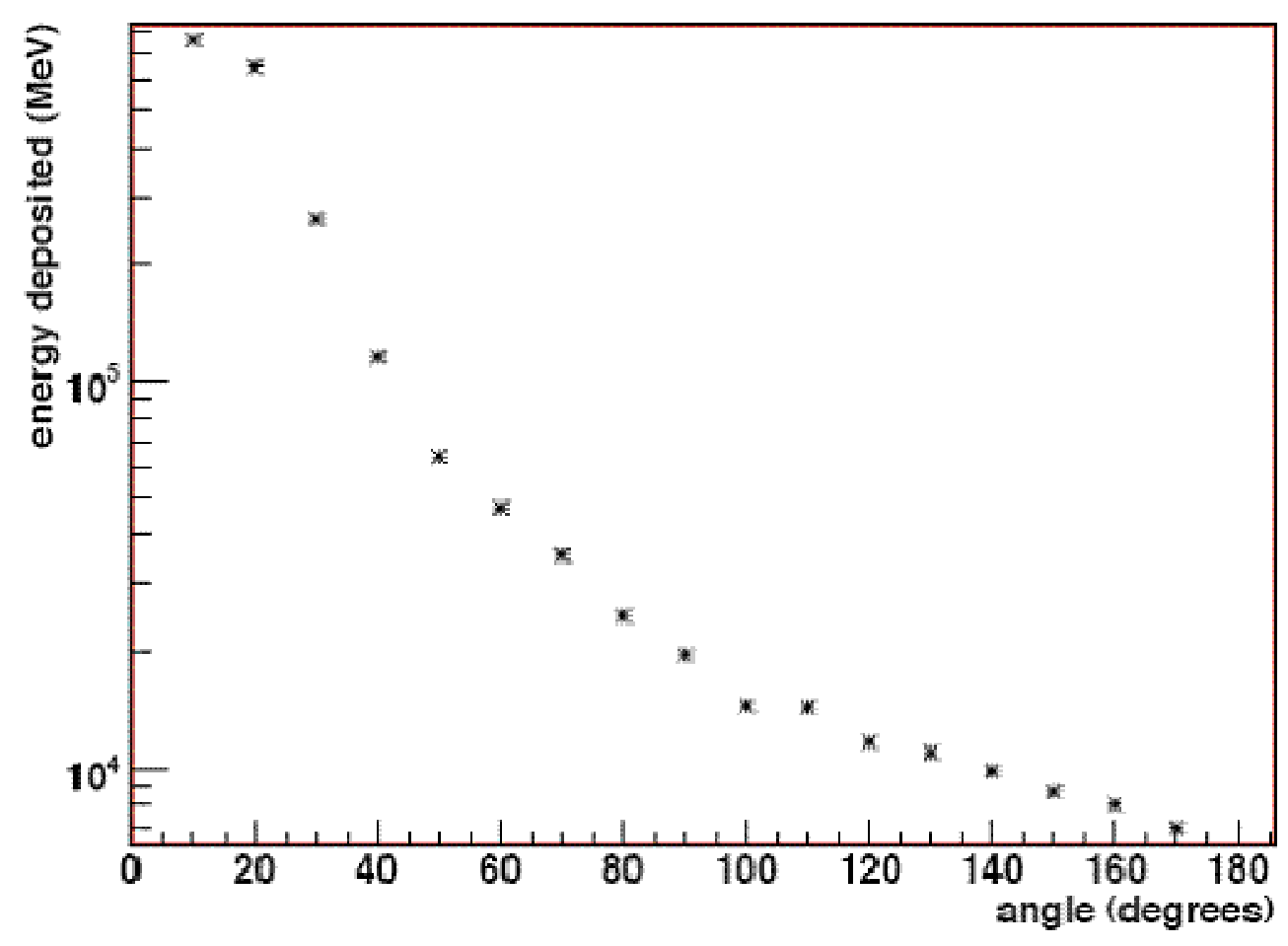

